\title{
Reduction PAPR of OFDM Signals by Combining Grouped DCT Precoding with PTS
}

\author{
Zhongpeng Wang1,2, Shoufa Chen 1 \\ ${ }^{1}$ School of Information and Electronic Engineering, Zhejiang University of Science and Technology, Hangzhou, \\ China \\ ${ }^{2}$ State Key Laboratory of Millimeter Waves, Southeast University, Nanjing, China \\ Email:wzp1966@sohu.com
}

Received 19 August 2014; revised 15 September 2014; accepted 7 October 2014

Copyright (C) 2014 by authors and Scientific Research Publishing Inc.

This work is licensed under the Creative Commons Attribution International License (CC BY).

http://creativecommons.org/licenses/by/4.0/

(c) (i) 0pen Access

\section{Abstract}

The high peak-to-average power ratio (PAPR) is one of the serious problems in the application of OFDM technology. In this paper, an improved partial transmit sequence (PTS) scheme based on combining the grouped discrete cosine transform (DCT) with PTS technique is proposed. In the proposed scheme, the adjacent partitioned data are firstly transformed by a DCT into new modified data. After that the proposed scheme utilizes the conventional PTS technique to further reduce the PAPR of the OFDM signal. The performance of the PAPR is evaluated using a computer simulation. The simulation results indicate that the proposed scheme may improve the PAPR performance compared with the conventional PTS scheme, the grouped DCT scheme, and original OFDM respectively.

\section{Keywords}

Orthogonal Frquency-Division Multiplexing (OFDM), Peak-to-Average Power Ratio (PAPR), Partial Transmit Sequence (PTS), Discrete Cosine Transform (DCT)

\section{Introduction}

Orthogonal frequency division multiplexing (OFDM) has been recently seen rising popularity in wireless applications. For wireless communications, an OFDM-based system can provide greater immunity to multi-path fading and reduce the complexity of equalizers [1] [2]. OFDM has been employed in many communication systems. However, the major drawback of OFDM signal is its large peak-to-average power ratio (PAPR), which causes poor power efficiency or serious performance degradation to transmit power amplifier.

Many techniques have been proposed to reduce the PAPR of OFDM signals. Such as clipping, coding, partial 
transmit sequence (PTS), selected mapping (SLM), interleaving, nonlinear companding transforms, hadamard transforms and other techniques etc. [3]-[5]. These schemes can mainly be categorized into signal scrambling techniques, such as PTS, and signal distortion techniques such as clipping. Among those PAPR reduction methods, the simplest scheme is to use the clipping process. However, using clipping process causes both in-band distortion and out-of-band distortion. Recently, the precoding technique has aroused the attention in reduction PAPR of OFDM signals. The precoding techniques show great promise as they are simple linear techniques to implement. The precoding method may reduce the PAPR of OFDM signal without signal distortion. The BER performance of the precoded OFDM system can be improved due to obtaining the frequency diversity in precoded systems. For example, the Hardamard precoding, DCT precoding, Hartley precoding have been employed in OFDM system [6] [7]. The SLM and PTS are distortion-less PAPR reduction techniques. PTS scheme is considered to be better than SLM. In PTS scheme, all the subcarriers are partitioned into multiple disjoint subblocks and then each of the sub-blocks are multiplied by a set of rotating phase factors such that the PAPR is reduced. However, the information about the phase factors needs to be conveyed to the receiver as the side information (SI). There is additional complexity due to the side information insertion.

In order to improve the PAPR performance of OFDM signal many schemes based on combing PTS or SLM and precoding have been proposed. For example, the precoding based Selected Mapping (PSLM) is proposed in literature [8]. The precoding technique is the efficient, signal independent, distortion-less and it does not require any optimization algorithm. In literatures [9], the grouped DCT precoding technique is proposed to reduce the PAPR of optical OFDM signal. In this paper, we present a grouped DCT precoding based PTS technique for PAPR reduction in OFDM systems. In the proposed system we applied DCT based precoder after the data partition and before the IFFT in the PTS based on OFDM (PTS-OFDM) system.

\section{OFDM Signal Model and PAPR Problem}

Consider an OFDM consisting of $\mathrm{N}$ subcarriers. Let a block of $N$ symbols $\mathbf{X}=\left\{X_{k}, k=0,1, \cdots, N-1\right\}$ is formed with each symbol modulating one of a set of subcarriers $\left\{f_{k}, k=0,1, \cdots, N-1\right\}$. the $N$ subcarriers are chosen to be orthogonal, that is, $f_{k}=k \Delta f$, where $\Delta f=1 /(N T)$ and $T$ is the original symbol period. Therefore, the complex baseband OFDM signal can be written as

$$
x(t)=\frac{1}{\sqrt{N}} \sum_{k=0}^{N-1} X_{k} \mathrm{e}^{j 2 \pi f_{k} t}, \quad 0 \leq t \leq N T
$$

In general, the PAPR of OFDM signals $x(t)$ is defined as the ratio period between the maximum instantaneous power and its average power during an OFDM symbol. It can be expressed as

$$
\text { PAPR }=\frac{\max _{0 \leq t \leq N T}\left[|x(t)|^{2}\right]}{1 /(N T) \int_{0}^{N T}|x(t)|^{2} \mathrm{~d} t}
$$

Reducing the $\max |x(t)|$ is the principle goal of PAPR reduction techniques. In practice, most systems deal with a discrete-time signal, therefore, we have to sample the continuous-time signal $x(t)$.

To better approximate the PAPR of continuous-time OFDM signals, the OFDM signals samples are obtained by $L$ times oversampling. By sampling $x(t)$ defined in Equation (1), at frequency $f_{s}=L / T$, where $L$ is the oversampling factor, the discrete-time OFDM symbol can be written as

$$
x(n)=\frac{1}{\sqrt{N}} \sum_{k=0}^{N-1} X_{k} \mathrm{e}^{j \frac{2 \pi}{N L} k n}, \quad 0 \leq n \leq N L-1
$$

The PAPR computed form the $L$-times oversampled time domain OFDM signal samples can be defined as

$$
\operatorname{PAPR}[x(n)]=\frac{\max _{0 \leq t \leq N L-1}\left[|x(n)|^{2}\right]}{E\left[|x(n)|^{2}\right]}
$$


The cumulative distribution function (CDF) is one of the most regularly used parameters, which is used to measure the efficiency of and PAPR technique. The CDF of the amplitude of a signal sample is given by

$$
F(z)=1-\exp (z)
$$

In many literatures, the complementary cumulative distribution (CCDF) is commonly used instead of the CDF to evaluate the PAPR of OFDM signals. The CCDF is given by

$$
P(\operatorname{PAPR}>z)=1-P(\operatorname{PAPR}<z)=1-(F(z))^{N}=1-(1-\exp (-z))^{N}
$$

\section{The Partial Transmit Sequence (PTS)}

The PTS technique was first described by Muller and Huber [11]. Figure 1 shows the block of the PTS technique. The input data block of $N$ symbols is firstly partitioned into smaller $V$ disjoint subblocks, which are represented by the vectors $\left\{\mathbf{X}^{(v)}, v=0,1, \cdots, V-1\right\}$, such that

$$
\mathbf{X}=\sum_{v=0}^{V-1} \mathbf{X}^{(v)}
$$

where $\mathbf{X}^{(v)}=\left[\begin{array}{llll}X_{0}^{(v)} & X_{1}^{(v)} & \cdots & X_{N-1}^{(v)}\end{array}\right]$ with $X_{k}^{(v)}=X_{k} \quad$ or $0 \quad(0 \leq v \leq V-1)$. The data for the every subcarrier in each sub-block is weighted by a phase factor. These phase factors are selected such that the PAPR of the combined signal is minimized. The objective of the PTS technique to find the optimum combination of factors for subblocks that minimizes the PAPR. Then the sub-blocks $X^{(v)}$ are transformed into $V$ time-domain partial transmit sequences

$$
\mathbf{x}^{(v)}=\left[\begin{array}{llll}
x_{0}^{(v)} & x_{1}^{(v)} & \cdots & x_{N-1}^{(v)}
\end{array}\right]=\operatorname{IFFT}\left(\mathbf{X}^{(v)}\right)
$$

These sub-blocks are independently rotated by phase factor $\mathbf{b}=\left\{b_{v}=\mathrm{e}^{j 2 \pi l / W} \mid l=0,1, \cdots, W-1\right\}$. Where $W$ is the number of allowed phase factors. Since we are dealing with the amplitude of the signal samples, one phase factor can be fixed without any performance loss, e.g., $b_{1}=0$. So we should perform exhaustive search for $W$ phase factors. Hence, $W^{V-1}$ sets of phase factors are searched to find the optimum set of phase factors. The search complexity increase exponentially with the number of subblocks $V$.

The time domain signal after combining is given by

$$
\tilde{\mathbf{x}}=\sum_{v=0}^{V-1} b_{v} \mathbf{x}^{(v)}
$$

The one with the minimum PAPR is chosen for transmission.

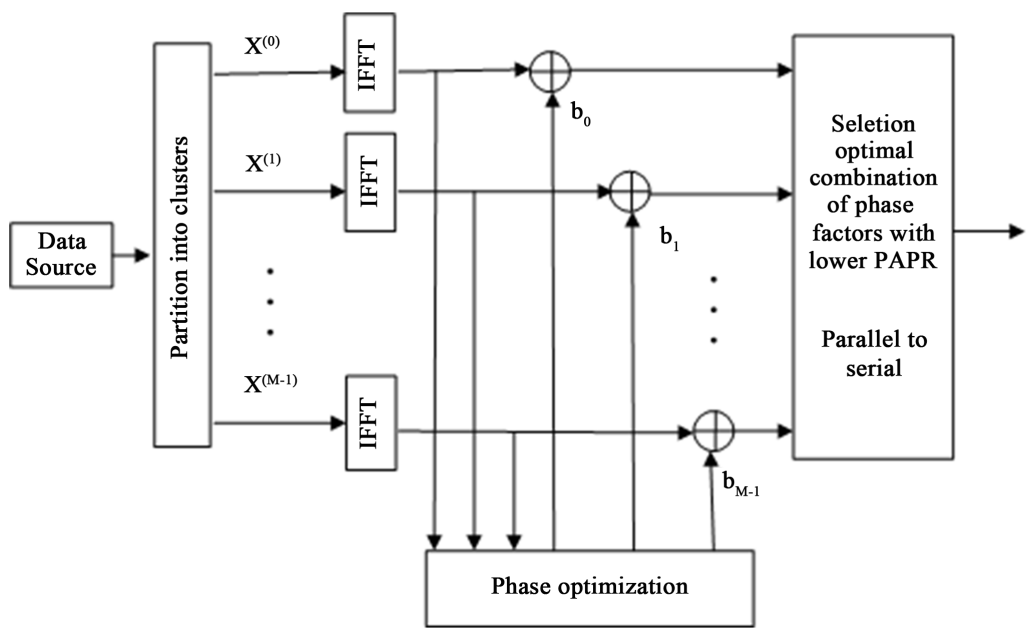

Figure 1. Block diagram of conventional PTS-OFDM scheme. 


\section{Grouped DCT Precoding}

The paper, by Park et al., [10] proposes a scheme for PAPR reduction in OFDM transmission using hadamard transform. Similar to the hadamard precoding, the discrete cosine transform (DCT) precoding is also applied to reduce the PAPR of OFDM signal. The application of this DCT matrix converts the time-domain signal into the new transform-domain signal. The DCT is an orthogonal linear transform that can be implemented by a butterfly structure, similar to the IFFT. DCT is a real transform in which the data are multiplied by a cosine function. The $M \times M \quad$ DCT matrix $\mathrm{P}$ is given as

$$
P_{i j}= \begin{cases}\frac{1}{\sqrt{M}} & i=0,0 \leq j \leq M-1 \\ \sqrt{(2 / M)} \cos \left[\frac{(2 j+1) i \pi}{2 M}\right] & 1 \leq i \leq M-1,0 \leq j \leq M-1\end{cases}
$$

where $i$ and $j$ represent the row and column entries, respectively. For a $1 \times M$ vector $X, Y$ indicates its DCT. Therefore, we obtain $Y=P X$, where $C$ is called the DCT matrix.

In Ref. [9], the grouped DCT precoding was presented in optical OFDM system. The grouped precoding based on grouped DCT reduces the computational complexity of precoding and inverse precoding. Figure 2 shows the block diagram of the grouped DCT precoding scheme. The main purpose of grouped precoding is to reduce the system complexity. The basic idea behind this approach is that the input data block of $N$ symbols is firstly partitioned into $V$ subblocks. Assume that $N=V M$. The $M$ is the number symbols of every subblock. After that the precoding matrix with a much smaller size is applied to each subblock.

\section{Proposed Scheme}

In order to reduce the PAPR of an OFDM signal, the combination of grouped DCT precoding and PTS can be used. The system block is showed in Figure 3. Subblock partitioning can be classified into three categories: adjacent, interleaved and pseudo random subblock partitioning [11]. Every subblock data is firstly transformed by precoding matrix. The output signal of the every precoder is represented by the vectors $\left\{\mathbf{Y}^{(v)}=\mathbf{P} \mathbf{X}^{(v)}, v=0,1, \cdots, V-1\right\}$, such that

$$
Y=\sum_{v=0}^{V-1} Y^{(v)}=\sum_{v=0}^{V-1} \mathbf{P} \mathbf{X}^{(v)}
$$

Then the subblocks $Y^{(v)}$ are transformed into $V$ time-domain partial transmit sequences

$$
y^{(v)}=\left[\begin{array}{llll}
y_{0}^{(v)} & y_{1}^{(v)} & \cdots & y_{N-1}^{(v)}
\end{array}\right]=\operatorname{IFFT}\left(Y^{(v)}\right)
$$

These sub-blocks are independently rotated by phase factor $\mathbf{b}=\left\{b_{v}=\mathrm{e}^{j \theta_{v}}, v=0,1, \cdots, V-1\right\}$. The time domain signal after combining is given by

$$
\tilde{x}=\sum_{v=0}^{V-1} b_{v} y^{(v)}
$$

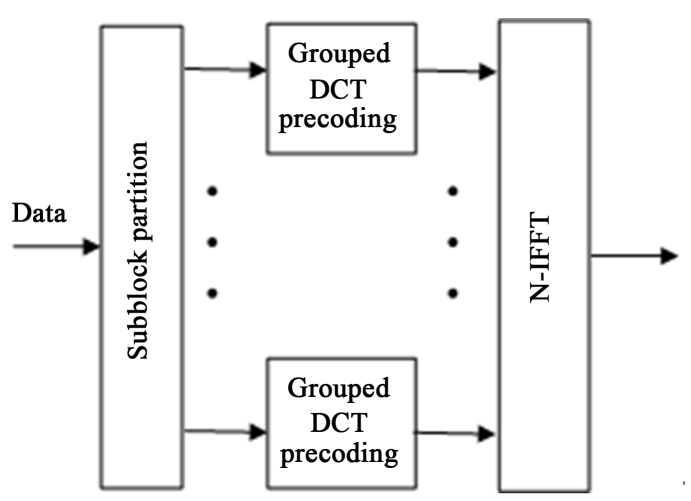

Figure 2. Block of the grouped DCT precoding scheme. 


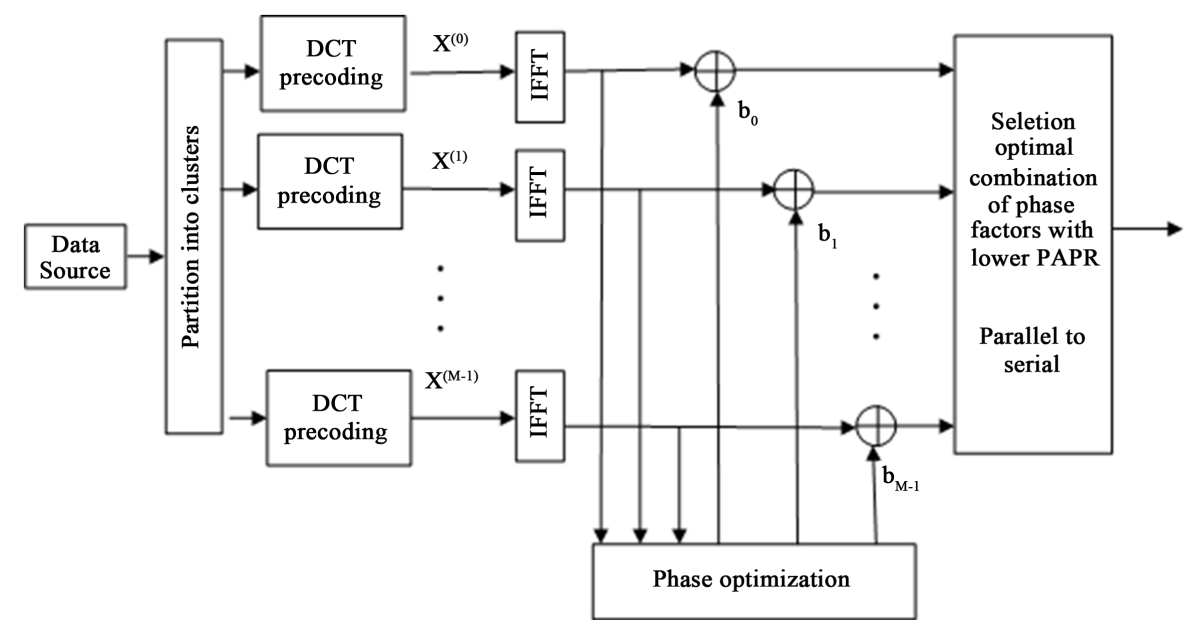

Figure 3. Block diagram of proposed combining grouped DCT and PTS OFDM scheme.

The vector $y^{(v)}$, called partial transmit sequence, is the IFFT of $Y^{(v)}$. The weighting factors are chosen to minimize the PAPR by searching for the appropriate combination of each cluster and by corresponding weighting factors.

$$
\left\{\tilde{b}^{(1)}, \tilde{b}^{(2)}, \cdots, \tilde{b}^{(V)}\right\}=\underset{\left\{b^{(1)}, b^{(2)}, \cdots, b^{(v)}\right\}}{\arg \min }\left(\max _{0 \leq n \leq N-1}\left|\sum_{v=1}^{V} b^{(v)} y_{n}^{(m)}\right|\right)
$$

The combination with weighting factors is called rotation factor or combining sequence. Optimized transmit sequence is

$$
\tilde{y}=\sum_{v=1}^{V} \tilde{b}^{(v)} y^{(v)}
$$

By exploiting the precoding in the first step the PAPR of OFDM signals may be effectively reduced. Moreover, the PTS in the second step further reduces the PAPR of OFDM signals.

\section{Simulation Results}

In this section, computer simulations are used to evaluate the peak-to-average ratio reduction capability with proposed scheme. The complement cumulative distribution function (CCDF) of the PAPR of OFDM signals is used to evaluate the performance of proposed scheme in comparison with other conventional reduction PAPR methods. In simulation, we assume an OFDM system with 64 subcarriers $(N=64)$ or 128 subcarriers $(N=128)$ with QPSK data symbols. The subcarriers are divided into 2 or 4 clusters (i.e. $V=2$, 4). Also assume that the number of allowed phase factors is $2(W=2)$ with $\mathbf{b}=\left\{\begin{array}{ll}1 & -1\end{array}\right\}$.

Figure 4 shows PAPR reduction performance of the grouped DCT precoding and the conventional PTS schemes with subcarriers of 128. The out two curves are the simulation results of the original OFDM and the grouped DCT precoded OFDM signals. The simulation results are obtained by the 50,000 OFDM blocks. We can see that the grouped DCT precoding scheme can obtain $1.5 \mathrm{~dB}$ PAPR reduction compared with the original OFDM system. Three inner curves describe the conventional PTS $(V=4)$. From the figure we can see the random partition may provide the best performance. The PAPR performance of the interleaving method is the worst.

Figure 5 shows the effect comparisons of the PAPR reduction performance of the proposed scheme with difference partition schemes. The sub-carrier number of OFDM signals is 128. Three kinds of sub-block partitioning methods are used in the proposed scheme. We can see that the PAPR performance of the proposed scheme with adjacent partition is the best.

Figure 6 shows the effect comparisons of the PAPR reduction performance of the proposed scheme with difference partition schemes. The subcarrier number of OFDM signals is 64. The similar results to the Figure 5 are obtained. We also can see that the PAPR performance of the proposed scheme with adjacent partition is the best.

Figure 7 shows the CCDF performance of the adjacent, interleaved and pseudo-random subblock partitioning 


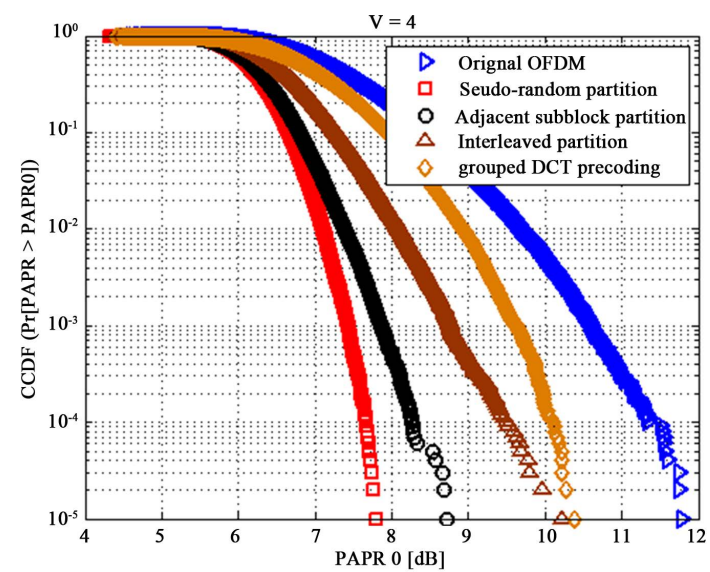

Figure 4. Comparison of the PAPR of the OFDM signals with different partition schemes, the grouped DCT precoded OFDM signal and original OFDM signal.

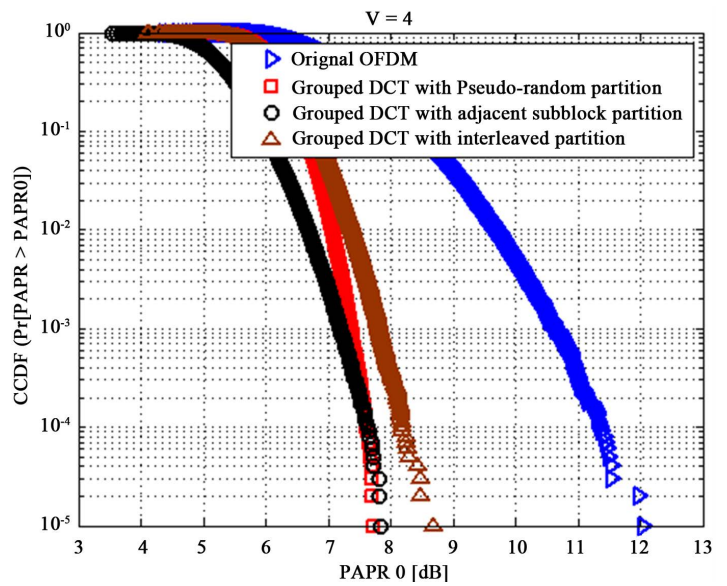

Figure 5. Comparison of the PAPR of the proposed grouped DCT precoding and PTS schemes with different partition methods at subcarriers $=128$.

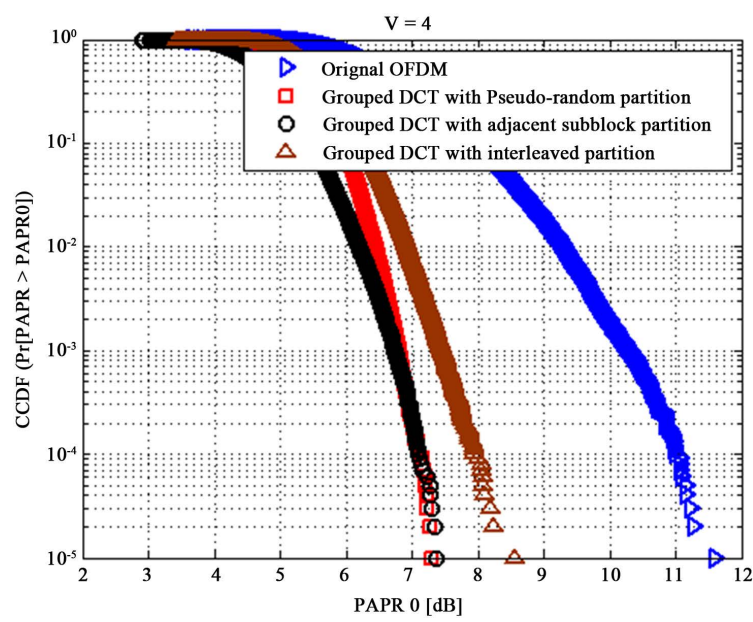

Figure 6. Comparison of the PAPR of the proposed grouped DCT precoding and PTS schemes with different partition methods at subcarriers $=64$. 


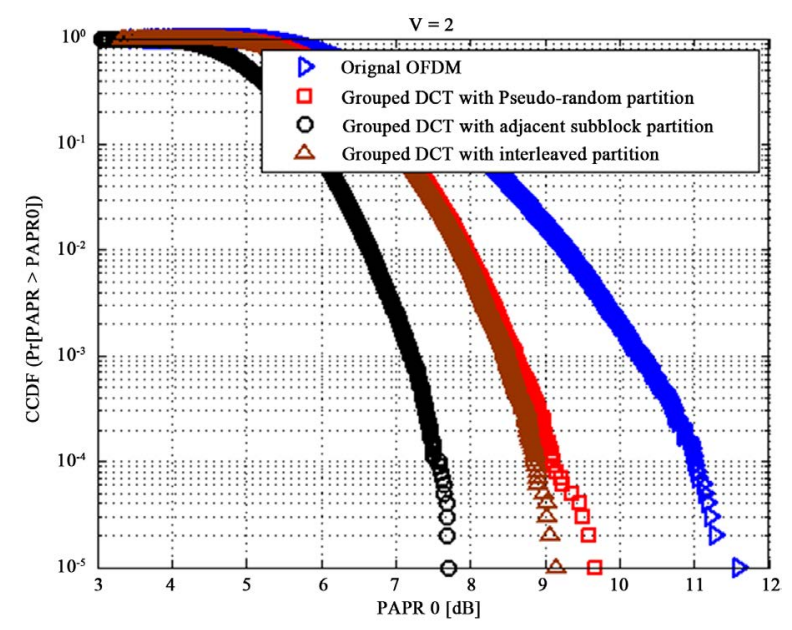

Figure 7. Comparison of the PAPR of the proposed grouped DCT precoding and PTS schemes with different partition methods at sub-blocks $=2$.

schemes with $V=2$. We also can see that the PAPR performance of the proposed scheme with adjacent partition is the best.

\section{Conclusion}

In this paper, a PAPR reduction scheme combing grouped DCT precoding and PTS technique is proposed. The PAPR reduction performances are evaluated by computer simulation. Simulation results state that the PAPR performance of the proposed scheme with adjacent partition is improved compared to original PTS, grouped DCT precoding, and original OFDM. At CCDF $=10^{-3}$, proposed scheme may obtain approximately $3.5 \mathrm{~dB}$ more PAPR reduction than the original OFDM system.

\section{Acknowledgements}

This work was supported in part by the by the Open Fund of the State Key Laboratory of Millimeter Waves (Southeast University, Ministry of Education, China) under K201214, by the Zhejiang Provincial Natural Science Foundation of China under LY13F050005, and by the key industrial project of Special major science and technology of Zhejiang Province under 2012C11016-2.

\section{References}

[1] Bahai, A.R. and Saltzberg, B.R. (1999) Multi-Carrier Digital Communications: Theory and Applications of OFDM. Kluwer Academic/Plenum Publishes, New York.

[2] Rohling, H., May, T., Bruninghaus, K. and Grunheid, R. (1999) Broadband OFDM Radio Transmission for Multimedia Applications. Proceeding of IEEE, 87, 1778-1789. http://dx.doi.org/10.1109/5.790637

[3] Jiang, T. and Imai, Y. (2008) An Overview: Peak-to-Average Power Ratio Reduction Techniques for OFDM Signals. IEEE Transaction on Broadcasting, 54, 257-268. http://dx.doi.org/10.1109/TBC.2008.915770

[4] Goela, A., Guptaa, P. and Agrawalb, M. (2013) SER Analysis of PTS Based Techniques for PAPR Reduction in OFDM Systems. Digital Signal Processing, 23, 302-313.

[5] Lain, J.-K., Wu, S.-Y. and Yang, P.-H. (2011) PAPR Reduction of OFDM Singals Using PTS: A Real-Valued Genetic Approach. Journal of Wireless Communications and Networking, 126.

[6] Ranjha, B. and Kavehrad, M. (2013) Precoding Techniques for PAPR Reduction in Asymmetrically Clipped OFDM Based Optical Wireless System. SPIE OPTO. International Society for Optics and Photonics, Article ID: 86450R.

[7] Sun, S.L., Hou, C.P. and Yan, L. (2008) A Novel Method Based DCT to Reduce PAPR of OFDM Systems. IEEE, 1-5.

[8] Jeoti, V. (2010) DCT Precoded SLM Technique for PAPR Reduction in OFDM Systems. International Conference on Intelligent and Advanced Systems (ICIAS 2010), Kuala, 15-17 June 2010.

[9] Wang, Z. and Zhang, S. (2013) Grouped DCT Precoding for PAPR Reduction in Optical Direct Detection OFDM Sys- 
tems. Optoelectronics Letters, 9, 213-216. http://dx.doi.org/10.1007/s11801-013-3021-7

[10] Park, M., Heeyong, J., Cho, N., Hong, D. and Kang, C. (2000) PAPR Reduction in OFDM Transmission Using Hadamard Transform. IEEE International Conference o Communications, 1, 430-433.

[11] Muller, S.H. and Huber, J.B. (1997) OFDM with Reduced Peak-to-Average Power Ratio by Optimum Combination of Partial Transmit Sequences. IEEE Electronics Letters, 33, 368-369. http://dx.doi.org/10.1049/el:19970266 
Scientific Research Publishing (SCIRP) is one of the largest Open Access journal publishers. It is currently publishing more than 200 open access, online, peer-reviewed journals covering a wide range of academic disciplines. SCIRP serves the worldwide academic communities and contributes to the progress and application of science with its publication.

Other selected journals from SCIRP are listed as below. Submit your manuscript to us via either submit@scirp.org or Online Submission Portal.
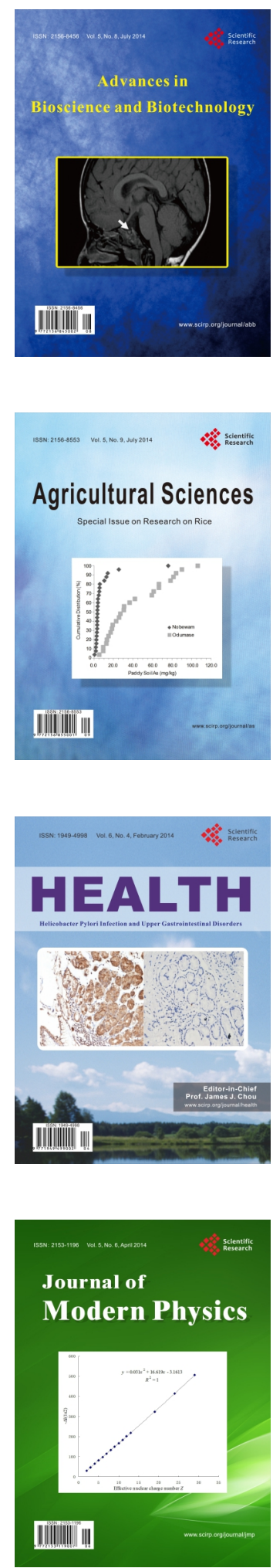
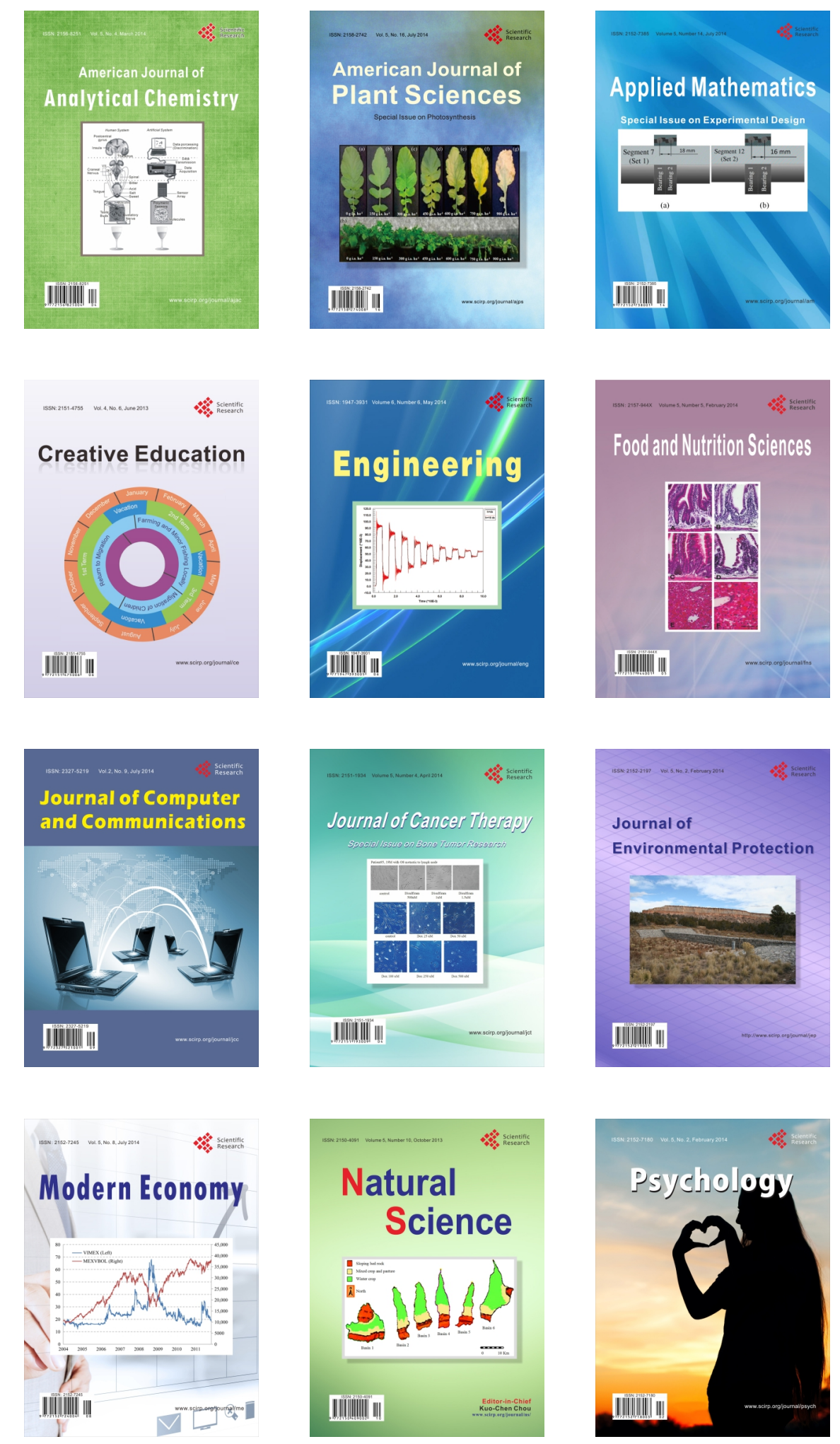